\title{
Linx
}

Revue des linguistes de l'université Paris X Nanterre

42 | 2000

Approches sociolinguistiques du plan phonique

\section{Les effets théoriques des pratiques de transcription}

Lorenza Mondada

\section{(2) OpenEdition}

\section{Journals}

Édition électronique

URL : http://journals.openedition.org/linx/902

DOI : $10.4000 /$ linx.902

ISSN : 2118-9692

\section{Éditeur}

Presses universitaires de Paris Nanterre

\section{Édition imprimée}

Date de publication : 1 juin 2000

Pagination : 131-146

ISSN : 0246-8743

\section{Référence électronique}

Lorenza Mondada, « Les effets théoriques des pratiques de transcription », Linx [En ligne], 42 | 2000,

mis en ligne le 02 juillet 2012, consulté le 19 avril 2019. URL : http://journals.openedition.org/linx/902 ; DOI : 10.4000/linx.902

Ce document a été généré automatiquement le 19 avril 2019.

Département de Sciences du langage, Université Paris Ouest 


\title{
Les effets théoriques des pratiques de transcription
}

\author{
Lorenza Mondada
}

\section{Les enjeux théoriques de la transcription}

1 Les problèmes de transcription ne sauraient être réduits à de simples questions techniques : cette position est aujourd'hui défendue par la plupart des auteurs qui thématisent les enjeux de la transcription. Depuis l'article pionnier d'Ochs (1979) on reconnaît en effet la dimension théorique des pratiques et des conventions de transcription. La transcription n'est pas simplement une activité sélective ${ }^{1}$, mais plus radicalement une entreprise interprétative - ce que retient l'expression de « halb-interpretative Arbeitstranskription » par laquelle Ehlich et Rehbein (1976) désignent leur propre système dès les années '70. Loin d'être un miroir plus ou moins fidèle de la langue parlée, la transcription et son système de notations sont donc "theory-laden ", dans la mesure où ils incorporent les présupposés théoriques du transcripteur dans les modes de représentation écrite de l'oral.

2 Si ces affirmations paraissent aujourd'hui incontestées ${ }^{2}$, elles continuent souvent à revêtir un caractère très général: les conséquences configurantes des choix des transcripteurs restent à expliciter et à décrire de façon précise. S'il n'existe pas de transcription «neutre » ou « objective », il reste que les choix possibles en la matière ne sont pas équivalents entre eux et impliquent - de façon souvent implicite - des positionnements spécifiques, à rapporter aux fins pratiques ${ }^{3}$ et théoriques poursuivies par l'analyste qui les adopte.

3 1.2. La transcription est un objet de réflexion pouvant intéresser non seulement la méthodologie et l'épistémologie de la linguistique, mais aussi une perspective inspirée de la sociologie des sciences, qui interroge la façon dont le linguiste fabrique ses données (Mondada, 1998). De ce point de vue, notre analyse de la transcription converge avec les analyses des pratiques et des techniques d'inscription visuelle dans les sciences naturelles (Latour, 1985 ; Lynch, 1988 ; Mondada, 1998, pt.3) : la transcription exploite les ressources 
de l'écrit pour produire une intelligibilité de l'oral fondée sur des opérations de filtrage des «bruits » ou d'autres aspects jugés non significatifs, de discrétisation du continuum sonore, d'homogénéisation dans le cadre de conventions systématiques. Ces processus contribuent à structurer l'oral en l'intégrant dans un ordre spatio-visuel et en en facilitant la saisie perceptive par l'œili'.

4 Le passage de l'oral à l'écrit effectué par la transcription a des conséquences configurantes pour le statut qu'y acquiert la langue parlée : le fait de réécouter de façon indéfiniment répétée des fragments d'oralité exerce un effet de loupe agrandissant des phénomènes qui autrement échappent à l'ouïe, les faisant littéralement émerger comme tels (Auer, 1993) ; la possibilité d'enregistrer d'abord et de transcrire ensuite a un effet de décontextualisation et d'extraction de l'oral par rapport à son contexte singulier de production (Franck, 1985) ; le fait de noter ces phénomènes a un effet de fixation du flux dynamique (Bergmann, 1985). En migrant du terrain à la bande puis de la bande à la page, l'oral se transforme donc radicalement ${ }^{5}$.

5 1.3. La description du lien entre les pratiques de la transcription et les présupposés théoriques de sa production et de son usage permet de poser un problème de circularité : de nombreuses interprétations de phénomènes se trouvent en effet incorporées dans la transcription a priori, alors qu'elles sont le but déclaré de l'analyse censée s'exercer a posteriori sur cette transcription. Ce problème se pose avec une acuité particulière par rapport à des objets hautement controversés ou à des phénomènes complexes articulant plusieurs dimensions; leur "naturalisation» dans une transcription qui les résoudrait avant même de les poser, en en réifiant le traitement dans des choix de notation, pose un problème majeur d'analyse.

6 C'est pourquoi nous insisterons sur l'importance de l'explicitation des choix matérialisés dans la transcription, notamment sur les perspectives qu'ils incarnent: perspective du transcripteur et du modèle théorique qu'il adopte, déterminant les choix de ce qu'il est ou non pertinent de noter ; perspective implicite échappant à la théorisation et rejoignant souvent le sens commun; perspective des locuteurs qui eux aussi s'orientent vers la pertinence de certaines formes et de certaines catégories dans le déroulement même de l'interaction.

7 1.4. Notre but ici est donc de montrer la façon dont les enjeux de transcription sont structurants par rapport à une série de problématiques controversées en sciences du langage. Ces enjeux seront explicités par rapport à des opérations fondamentales qui traversent la pratique de la transcription et qui consistent à traiter des formes en les discrétisant, en les découpant, en les identifiant et en les catégorisant ${ }^{6}$. Ce traitement analytique des formes incorporé dans les choix de transcription contribue de façon centrale à rendre descriptible, analysable et interprétable le flux oral enregistré.

8 Pour ce faire, nous nous pencherons sur trois problèmes particuliers, dont l'émergence et la résolution dépendent, et en retour éclairent, de nombreuses questions de transcription : le problème de la discrétisation, de l'identification et de la catégorisation des unités de la parole orale (2.), celui de la notation plus ou moins analytique et articulée de certains phénomènes, comme la qualité de la voix ou le rire (3.), celui de la reconnaissabilité et de la catégorisation des formes linguistiques, voire des langues en présence (4.), mise en évidence notamment dans les transcriptions d'interactions plurilingues. Ces phénomènes ont en commun de s'inscrire dans des problématiques où se confrontent des modèles divergents et de montrer le lien étroit entre transcription d'une part et discrétisation, segmentation, catégorisation des formes de l'oral de l'autre. 


\section{La discrétisation des unités de l'oral} forme de traitement : les opérations pratiques de découpage mot à mot, de segmentation, de délimitation de la chaîne parlée posent d'emblée la question théorique des unités pertinentes de l'oral.

parlée dans la critique d'unités typiquement liées à l'écrit standard, comme la phrase, et dans la proposition de nouvelles unités, plus respectueuses des spécificités de l'oral. La controverse des unités ${ }^{7}$ n'est toutefois pas terminée, comme le montre la prolifération des solutions alternatives - de la "période" proposée par Berrendonner \& Reichler (1989), au "paragraphe » proposé par Morel \& Danon-Boileau (1998); des «units of cognitive information » de Gumperz \& Berenz (1993) aux «intonation units » de Chafe (1994), et, dans la littérature interactionniste, du «tour» aux "turn constructional units» (Sacks, Schegloff, Jefferson, 1974; Schegloff, 1996) - sans compter la prise en considération de la re-spécification de ces unités par les locuteurs eux-mêmes dans une perspective émique (Mondada, 2000b, pt. 4).

11 Cette controverse peut être approchée à travers l'observation de la façon dont la spatialisation des transcriptions produit des effets de découpage, voire de la façon dont certains auteurs « incorporent » leur définition des unités dans la segmentation de leur transcription.

D'une part, certaines conventions de transcription prévoient des signes indiquant explicitement le début et la fin des unités. C'est le cas des notations établies dans le cadre $\mathrm{du}$ projet ESF sur l'acquisition des langues par des adultes migrants, où figurent des « sense-units » ou « T-units » : « if the transcriber feels that he can unambiguously isolate a sense-unit (defined here intuitively as a sequence which expresses a complete thought or idea) he may enclose the sequence in @ @ " (Perdue, 1982: 261). Les unités sont ici définies selon un critère thématique : il n'est pas surprenant que leur découpage fasse recours à l'intuition, cette dimension étant connue dans la littérature comme posant d'énormes problèmes de définition conceptuelle et formelle d'entités thématiques explicitement reconnaissables.

13 D'autre part, le marquage des unités n'est pas toujours confié à un signe spécifique, mais est lié à la matérialité même de l'inscription écrite : la transcription s'organise en lignes et en paragraphes en dessinant ainsi des unités typographiques visuelles qui peuvent être investies d'une valeur théorique. Ainsi par exemple Chafe (1993) fait correspondre à chaque paragraphe une unité :

\begin{tabular}{|l|l|l|}
\hline (1) & $(\text { Chafe, 1993: 38 })^{8}$ & \\
\hline & $\begin{array}{l}\text { It is useful to differentiate substantive, regulatory, and fragmentary intonation units in a } \\
\text { transcription. One way is to add annotation S, R, or F after the designation of the speaker: }\end{array}$ \\
\hline & {$[\ldots .]$.} & \\
\hline & (8.1) (A) (F) & ... And so in $\wedge^{\wedge}$ between the-- \\
\hline
\end{tabular}




\begin{tabular}{|c|c|}
\hline$(8.2)(\mathrm{A})(\mathrm{S})$ & $\begin{array}{l}. . \text { okay the }{ }^{\wedge} \text { first two } \wedge^{\wedge} \text { rooms } \mid \text { are at the } \mathrm{t} \\
. . \text { at the }\end{array}$ \\
\hline &..$^{\wedge} \wedge$ front $\wedge^{\wedge}$ part of the ${ }^{\wedge}$ hall, \\
\hline (8.3) (B) (R) & ... Mhm, \\
\hline$(8.4)(\mathrm{A})(\mathrm{S})$ & $\begin{array}{l}. . \text { and so } . . \wedge^{\wedge} \text { between those } \ldots \text { the } \\
\wedge \text { entrances to those }\end{array}$ \\
\hline & $\begin{array}{l}\wedge \text { rooms and the }{ }^{\wedge} \text { bathroom } \mid \text { there's a } \\
\wedge^{\prime} \mathrm{lo}=\text { ng stretch of }\end{array}$ \\
\hline & $\wedge^{\wedge}$ hallway. \\
\hline
\end{tabular}

14 La transcription incorpore ainsi une analyse qui segmente le corpus en unités de base, les « intonation units » et les catégorise en types différents.

15 Face à cette mise en correspondance explicite entre unité typographique et unité définie dans un modèle théorique, d'autres usages n'attribuent aucune valeur explicite à la disposition visuelle de la transcription, sans échapper à un effet de découpage en unités. Ainsi en est-il toujours du paragraphe qui, chez d'autres transcripteurs, tend à correspondre au tour de parole :

\begin{tabular}{|l|l|}
\hline (2) & (Brès, 1995: 293) \\
\hline & A.2 - un des grands moments ç'a été : bien entendu le : / le mur \\
\hline & C.3 - raconte-nous ça tu veux ? \\
\hline & A.4 - (rire de A) François a dû le raconter \\
\hline & $\begin{array}{l}\text { C.5 - ah oui oui mais : on tient absolument à (2) ton (2) récit du mur tu n'y échapperas pas } \\
\text { (rire de C) }\end{array}$ \\
\hline
\end{tabular}

16 Dans ce protocole d'accord - et c'est une exception - la vérification (A.4) intervient après la requête (C.3): comme une façon de la refuser. L'enchaînement C.3/A.4 pose explicitement que la présence de narrations antérieures bloque l'actuelle possible mise en récit : A l'utilise comme argument.

17 La fin du paragraphe (repère typographique) correspond au passage de la parole à un autre locuteur (critère interactionnel) - chaque paragraphe étant en principe spécialisé pour les interventions d'un locuteur ${ }^{9}$. Or cette dernière unité est souvent davantage l'effet d'une disposition spatio-visuelle qu'une unité théorisée et revendiquée comme telle : ainsi dans notre exemple l'analyse ne fait aucune référence à une unité comme le « tour de parole » pour la désigner, puisque d'autres catégorisations des segments repérés sont utilisées (" vérification », " requête ", « enchaînement ») ${ }^{10}$.

18 Par ailleurs l'identification du paragraphe avec le «tour de parole » pose elle aussi des problèmes, exhibés par la notation des bribes en chevauchement - par lesquelles un 
locuteur tente de prendre la parole mais ne la prend pas -, des continuateurs (comme « mhm ») qui ne constituent pas une prise de parole, ou des tours qui sont commencés par un locuteur et terminés par un autre. Ces phénomènes, qui font l'objet d'analyses contradictoires dans la littérature, demandent une analyse des unités conversationnelles qui prenne en compte les postures de «locuteur en train de parler», de «locuteur entrant en compétition pour le tour ", de « locuteur qui s'apprête à prendre la parole » ou d'« auditeur attentif», où le fait de considérer le «tour » comme une unité pertinente ou non, de le traiter comme une unité structurelle ou comme un accomplissement pratique, de le définir comme une unité minimale ou comme une entité articulée en « unités de construction du tour » déclenche des interprétations très différentes des lignes ou des paragraphes de la transcription ${ }^{11}$.

C'est pourquoi une solution alternative consiste à traiter la ligne comme une unité non théorique mais purement graphique, en la faisant correspondre à un segment arbitrairement délimité par les marges disponibles de la page. Les transcriptions «en partition" (que Edwards, 1993: 11 oppose aux transcriptions "verticales " que nous venons de discuter) sont une solution qui ne décide pas du statut des segments produits par les locuteurs en succession ou en chevauchement et qui suspend l'interprétation séquentielle du déroulement de l'interaction. En voici un exemple :

\begin{tabular}{|c|c|}
\hline \multirow[t]{2}{*}{ (3) } & (Dausendschön-Gay, Gülich, Krafft, $1992: 242$ ) \\
\hline & 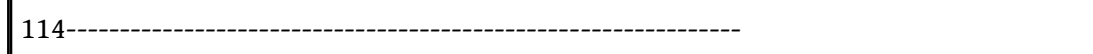 \\
\hline \multicolumn{2}{|r|}{ An : $\quad$ ah OUAIS ça je sais' ${ }^{*}$ mais je : } \\
\hline \multicolumn{2}{|r|}{ Do : c'est =un terme technique, + on l'a défini * (bas) à tu } \\
\hline & 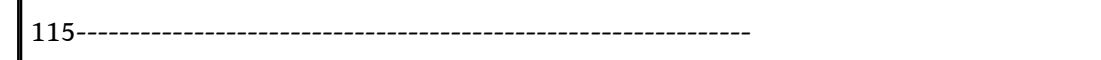 \\
\hline \multicolumn{2}{|r|}{ An: (pensive) ah, } \\
\hline \multicolumn{2}{|r|}{ Do : sais hein + .. ou tout un eh/ ah : tout simplement (bas) } \\
\hline \multicolumn{2}{|r|}{ 116----------------------------------------------------------------- } \\
\hline & An : ouais' \\
\hline & Do : un $:: e+.($ distinctement $)$ perturbaTION + de la communication" c'est mIEUx \\
\hline \multicolumn{2}{|r|}{ 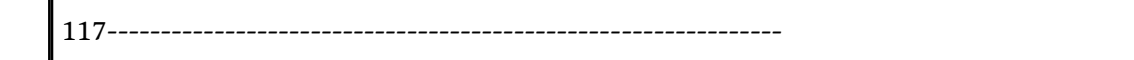 } \\
\hline \multicolumn{2}{|r|}{ An : $j(e)$ trouve que c'est mIEUx là, } \\
\hline \multicolumn{2}{|r|}{ Do: non' qui ont co :mme sourc : :e . comme eh/ (re- } \\
\hline \multicolumn{2}{|r|}{ 118------------------------------------------------------------------ } \\
\hline \multicolumn{2}{|r|}{ An : } \\
\hline & Do : lit très vite) dénomi/ ( ?. .) + une . per/ . per.tur.ba.TION’ . de LA, \& \\
\hline
\end{tabular}




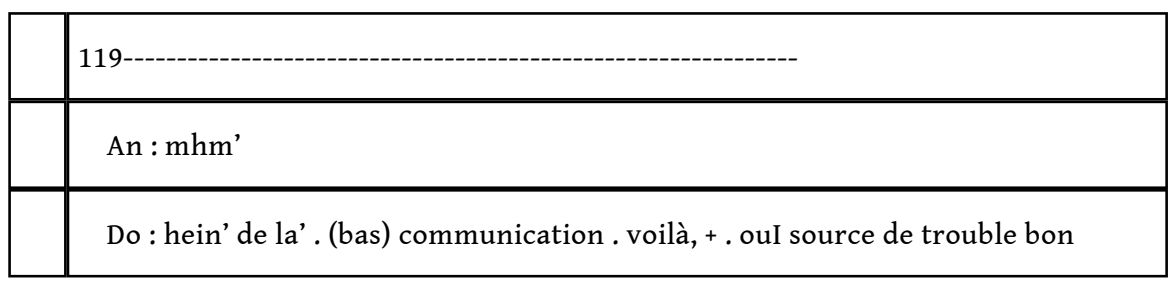

La comparaison des exemples 1-3 montre que la numérotation joue un rôle très important dans la production ou dans le déjouement de ces " effets d'unité » : numéroter les portées comme ci-dessus, ou bien les lignes ou les paragraphes, référer à la «ligne 5 » ou au «tour 5 » a des implications fondamentales pour les problèmes soulevés ici. Ceci montre bien le lien étroit existant entre décisions liées à la discrétisation et options de catégorisation des unités.

\section{Transcrire ou décrire?}

21 Les choix concernant le découpage analytique de la chaîne parlée ne concernent pas uniquement des segments tels que la période, l'unité intonative ou le tour : ils concernent plus généralement toute forme que le transcripteur isole et identifie dans le flux de l'oral. Les décisions prises par les transcripteurs exhibent leur conception de certains phénomènes oraux comme plus ou moins analysables, plus ou moins holistiquement interprétables, plus ou moins typifiables ${ }^{12}$.

De ce point de vue, les commentaires des transcripteurs, figurant souvent entre parenthèses, sont significatifs. Ils renvoient en effet à des phénomènes qui ne sont pas transcrits mais décrits. La description du phénomène renonce alors à appliquer les conventions de notations pour le représenter et exhibe ainsi les limites du système de transcription adopté, tout en les dépassant par la proposition d'une description qui considère le phénomène dans sa globalité. En voici un exemple :

\begin{tabular}{|c|c|c|}
\hline \multirow[t]{2}{*}{ (4) } & \multicolumn{2}{|c|}{ (Schmale, $1987: 220)$} \\
\hline & $1 \mathrm{~V}:$ & $(($ en chantant $))$ la bonne humeu :r- + \\
\hline & $2 \mathrm{I}:$ & (bas, peu sûre) bonne humeur- + \\
\hline & $3 \mathrm{~V}:$ & [tu sais' mais si, . gute Laune, Leu/ Laune, \\
\hline & $4 \mathrm{I}:$ & {$[$ non, ah, \& ((avec } \\
\hline & 5 & une grosse voix, autoritaire)) tu ne paRles pas d'allemand, + \\
\hline & $6 \mathrm{~V}:$ & $(($ en chuchotant $))$ parce que c'est (interdit ?) .+ \\
\hline & $7 \mathrm{I}:$ & [je n'aime pas, . zut, zet pareille, /pre/, \\
\hline & $8 \mathrm{~V}:$ & 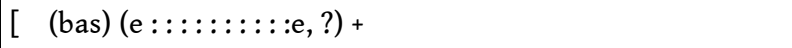 \\
\hline
\end{tabular}


Les conventions adoptées opposent la parenthèse simple pour les «Charakterisierungen der Sprechweise » et la double parenthèse pour les «subjektive Charakterisierungen des Transkribenten", en montrant par là même les enjeux interprétatifs de ce type de notation - même si leur distinction n'apparaît pas très tranchée (on peut se demander pourquoi choisir les parenthèses doubles au lieu des simples, par exemple à la ligne 2 où l'évaluation de l'assurance du locuteur est au moins aussi « subjective » que la description du ton de sa voix, chanté ou chuchoté, des lignes 1 et 6$)^{13}$. Alors que Schmale essaie d'approfondir la notation des commentaires ${ }^{14}$, Jefferson (1985) dans son article classique sur le rire explore et justifie l'intérêt d'approfondir la notation transcrite du phénomène. Devant transcrire une blague, elle oppose deux solutions :

\begin{tabular}{|l|l|l|}
\hline (5) & (Jefferson, 1985) \\
\hline & solution 1 & \\
\hline & Louise: & ((through bubbling laughter) ) Playing with his organ yeah \\
\hline & solution 2 & \\
\hline & Roger: & [Oh:. [hehh[h a h 'he:h]'heh \\
\hline & Louise : & [heh huh 'hh PLAYN(h)W(h)IZ O(h)R'N ya :h \\
\hline
\end{tabular}

Le rire est souvent transcrit entre parenthèses. Cette solution a des effets forts de typification et d'homogénéisation du phénomène. Elle est plus ou moins adaptée au type de rire : il y a des rires ponctuels, précisément localisés dans le flux de la parole orale, mais il y a aussi des rires qui se prolongent, qui investissent la parole et la déforment. En outre, il y a des situations où le rire est particulièrement pertinent pour l'activité en cours - ici il s'agit d'une blague grivoise où l'allusion sexuelle constitue la chute et correspond à l'avènement du rire : la forme particulière du rire est une ressource pour l'accomplissement de cette activité. Il est donc fondamental de recourir à une tra nscription qui en tienne compte - tout en reconnaissant que la transcription reste une tentative de représentation ${ }^{15}$. Décrire ce rire, au contraire, aurait comme effet de gommer les détails qui permettent précisément l'analyse de ce qui se passe ${ }^{16}$.

\section{La catégorisation des formes linguistiques}

Les effets discrétisants de la transcription s'exercent donc à différents niveaux de l'analyse linguistique et discursive : nous avons souligné celui des unités de l'oral et celui de phénomènes complexes échappant aux conventions ordinaires ; nous nous arrêterons ici sur celui des formes linguistiques elles-mêmes, le passage à l'écrit entraînant une décomposition analytique de la chaîne parlée en morphèmes et lexèmes souvent découpés et identifiés de façon tranchée par les conventions orthographiques et typographiques (cf. Duranti, 1997 : 124sv). Ces formes ne sont pas seulement identifiées, mais aussi catégorisées - comme appartenant au standard ou à une de ses variantes, comme compréhensibles ou non, comme s'inscrivant dans le système d'une langue ou 
pas, etc. Nous avons montré ailleurs que le linguiste n'est pas le seul à procéder à ces catégorisations, puisque les locuteurs eux-mêmes catégorisent les formes qu'ils énoncent ou qu'ils entendent et produisent ainsi une catégorisation vernaculaire des variétés, des registres, des répertoires, voire des langues elles-mêmes, dont l'étude est susceptible de bouleverser plus d'une typologie linguistique ou sociolinguistique (Mondada, 2000a, 2000c). Nous allons brièvement montrer ici que ces enjeux émergent dès la transcription que le linguiste fait de l'oral des locuteurs.

\subsection{Plus ou moins grandes indéterminations formelles et catégorielles}

Le travail du transcripteur consiste à isoler, à découper, à identifier, à rendre reconnaissables de façon écrite et tranchée les formes enregistrées. Ce travail peut entrer en contradiction avec le fait que parfois les formes ne sont ni produites ni reçues comme clairement reconnaissables, mais qu'elles possèdent un caractère indéterminé qui ne nuit pas nécessairement à la communication, et peut même la servir. Il peut alors être intéressant pour le linguiste de préserver cette indétermination pour en faire un objet d'étude.

Certains systèmes de transcription prévoient plusieurs possibilités de noter une forme indéterminée. Ainsi le système de transcription de l'exemple (4) cité plus haut prévoit plusieurs possibilités de traiter des formes non-standard:

\begin{tabular}{|l|l|l|}
\hline (6) & \multicolumn{2}{|l|}{ Extrait des conventions de Schmale (1987) (cf. 4) : } \\
\hline & /pre/ & phonemische Transkription \\
\hline & paRles & Grossschreibung von Lauten zeigt Abweichungen \\
\hline & & von der Standardaussprache \\
\hline & (interdit??) & transkribierter Wortlaut nicht absolut sicher (ein \\
\hline & & Fragezeichnen), sehr zweifelhaft (zwei Fragezeichen) \\
\hline
\end{tabular}

Ainsi à la ligne 7 de l'extrait 4 «/pre/» note une forme exhibée comme non reconnaissable, donc transcrite en API, alors que «zet pareille» note une forme reconnue, représentée par un trucage orthographique et par un marquage de la prononciation finale non standard; à la ligne 8 , « (e::::::::::e,?)» le signe interrogatif indique une forme marquée par le doute.

Ces conventions montrent qu'il est possible de distribuer les formes transcrites dans un espace catégoriel complexe, où elles sont catégorisées comme plus ou moins compréhensibles (transparentes ou opaques), comme plus ou moins normées (selon leur distance du standard), comme pouvant être plus ou moins associées à un pattern typifiable, attribuable à une catégorie sociale, etc. En particulier, le recours à la transcription $\mathrm{AP}^{17}$ permet de ne pas choisir l'orthographe de la langue dans laquelle on veut transcrire une forme, et donc de ne pas l'attribuer à une langue précise - mais a aussi comme effet de détacher visuellement ce qui est ainsi transcrit et de le rendre 
« étrange » par rapport au reste ; la notation de degrés d'incertitude (ainsi que la pratique de la multi-transcription) permet d'attribuer l'indécision aux transcripteurs (ou à la qualité de l'enregistrement) au lieu d'en faire une caractéristique de la forme elle-même ; finalement la notation de la forme comme incompréhensible (souvent xxx, où le nombre de $x$ renvoie au nombre de syllabes ou à la longueur approximative du segment) permet de ne pas se prononcer du tout sur elle. Il est donc possible de donner des représentations très différentes d'une forme, qui interviendront dans l'analyse de façon cruciale.

La variation des formes est parmi les aspects les plus difficiles à transcrire, tant les effets de catégorisation provoqués par les différentes solutions techniques sont importants. On peut replacer dans ce contexte la controverse sur ce que Blanche-Benveniste et Jeanjean (1987) appellent les "trucages orthographiques ", qu'elles dénoncent à cause de leurs effets de catégorisation stigmatisante, induisant des interprétations de l'oral ainsi transcrit comme relevant des catégories "populaire ", " peu éduqué ", "subalterne ", etc. Gumperz et Berenz (1993: 96-7) souscrivent à cette analyse, en soulignant les effets stéréotypisants de l'«eye dialect", tout en reconnaissant aussi la pertinence des informations qu'une telle transcription, lorsqu'elle est maîtrisée, peut apporter quant à la catégorisation du contexte par les participants eux-mêmes (comme «formel» ou « informel » par exemple) et en définitive quant à l'interprétation de l'interaction dans sa globalité.

\subsection{Transcriptions d'échanges plurilingues : le traitement des formes hybrides et des mixed languages}

Les échanges exolingues - marqués par une dissymétrie de compétences dans la langue de l'échange, reconnue par les participants eux-mêmes - et bilingues sont caractérisés par la présence de formes dont l'appartenance catégorielle est difficile à déterminer, étant souvent spécifique (appartenant à l'interlangue des non-natifs), mixte, sous-déterminée, ou neutralisée, donc indécidable. Ces caractéristiques, ainsi que les méthodes par lesquelles les participants procèdent eux-mêmes à leur catégorisation située, peuvent devenir un objet d'étude pour le linguiste à condition qu'elles soient préservées dans la transcription.

33 Alors que certaines notations visent à maintenir une certaine indétermination de la forme et à modaliser, voire à suspendre, leur identification catégorielle (cf. 4.1), d'autres modes de transcription visent à éliminer le plus possible les indécisions et/ou les variations. La notation ESF des transcriptions d'échanges avec des non-natifs va dans ce sens. En voici un exemple :

\begin{tabular}{|l|l|l|}
\hline (7) & (Corpus ESF ; cité in Vasseur, $1993: 47)$ \\
\hline & G : & $* y^{*}\left[\right.$ se le vestjur la] ${ }^{*}$ el ves / come se dice ? el vestir* hm? \\
\hline & N : & ah oui ils étaient ils étaient déguisés ils étaient \\
\hline & G: & *si $^{*}$ \\
\hline & N : & oui \\
\hline
\end{tabular}




\begin{tabular}{|c|c|}
\hline$G$ : & de [komo se di] *en frances ?* hm [se] un *insecto* hm + \\
\hline & *abeja* [se le vestjur] de andres *de la abeja* [no kompran pa ?] \\
\hline $\mathrm{N}$ : & non \\
\hline$G$ : & [komo se di ?] \\
\hline $\mathrm{N}$ : & un insecte tu dis? \\
\hline G : & *si que es la abeja* \\
\hline $\mathrm{N}:$ & ah oui je sais pas qu'est-ce que c'est? qu'est-ce qu'il fait? \\
\hline & uelle couleur il est? \\
\hline G : & *del del del animal* [ke ke ke dona] *la miel* \\
\hline $\mathrm{N}:$ & l'abeille \\
\hline : & l'abeille oui l'abeille ah oui \\
\hline
\end{tabular}

Ce système de transcription distingue les formes notées entre astérisques appartenant à la L1 du non-natif, des formes transcrites en API, entre parenthèses carrées, correspondant à l'interlangue du non-natif (et en l'occurrence à des éléments non standard de la langue cible). Cette distinction relève des catégories exogènes du linguiste ; elle ne rend pas compte du statut que ces formes ont pour les interlocuteurs, qui peuvent les traiter comme compréhensibles (c'est ce que fait $\mathrm{N}$ du premier tour de parole) ou comme opaques (c'est le cas ensuite d'« abeja »). Par ailleurs ces distinctions catégorielles n'excluent pas des hésitations du transcripteur: on trouve dans le même article des formes parfois transcrites de deux façons différentes - comme « *como se* [di]» (p. 50), qui segmente et catégorise différemment le « [komo se di] » de l'extrait cité; ou bien « *se preparan sobre el examen final por* [arribar] *al liceo* (p. 48) et «*el otro/ la cla/ del quinto para* après *arribar al* lycée " (p. 49), qui montrent une indécision entre l'attribution du verbe à la L1 ou à l'interlangue. Ces indécisions peuvent être la trace d'un phénomène intéressant, qui risque toutefois d'être gommé, rendu invisible, par des conventions imposant trop de choix catégorisants au transcripteur.

Une question analogue est posée par le traitement des phénomènes de code-switching qui repose sur la description souvent problématique des ressources linguistiques alternées, combinées voire bricolées par les interlocuteurs. Ces ressources sont catégorisées de différentes façons, selon le modèle utilisé : on distinguera par exemple entre des systèmes linguistiques différents convoqués successivement ${ }^{18}$, ou bien entre « matrix language » et « embedded language ». Ces distinctions sont bien représentées dans les transcriptions par le recours au marquage de chaque langue par une police de caractères spécifique.

Ce type de marquage se heurte cependant à des formes qui ne sont pas toujours attribuables à des grammaires clairement distinctes - qu'elles soient des hybrides, des mélanges inextricables qui ne peuvent être rapportés à deux langues originaires ou des formes indéterminées neutralisant des différences qui permettraient de tracer la 
frontière entre deux langues. Ce genre de phénomène montre l'intérêt que peuvent revêtir d'autres formes de transcription, ne codant pas d'emblée l'appartenance des formes en jeu. Plus radicalement, le choix des conventions de transcription semble être fortement lié à la théorie du code-switching défendue, au point de distinguer les modèles qui considèrent que le parler bilingue est descriptible comme la co-présence de deux langues qui se combinent tout en maintenant une autonomie de leurs systèmes respectifs, de ceux qui considèrent que le parler bilingue exploite un répertoire spécifique irréductible à l'une ou l'autre grammaire.

Dans l'article classique de Poplack (1980) sur le code-switching entre anglais et espagnol, la transcription distingue typographiquement les deux langues (au moyen de l'alternance entre le romain et les capitales), en utilisant systématiquement le romain pour la langue de base. Cette convention n'est toutefois pas explicitée comme telle et ne fait l'objet d'aucun commentaire - alors que l'article thématise les modes de codage du corpus pour ce qui est des catégories syntaxiques ayant servi à l'annoter (1980: 596sv). Le codage typographique n'est donc pas considéré comme une opération à expliciter, bien qu'il incorpore des décisions importantes. En voici un exemple :

\begin{tabular}{|l|l|l|}
\hline (8) & (Poplack, $1980: 598)$ \\
\hline & A : & Lo pusieron un ... ¿ ¿ómo se dice ? ¿un tutone? \\
\hline & & (They gave him a - how do you call it? tuton?) \\
\hline & B : & TUTOR ? \\
\hline
\end{tabular}

L'espagnol est désigné comme étant la langue de base, sans que cette décision soit motivée et puisse être évaluée par le lecteur, qui n'a accès qu'à deux tours de parole de l'échange.

On remarquera que la transcription de l'espagnol n'adopte pas uniquement l'orthographe de cette langue, mais aussi la ponctuation écrite qui lui est propre (en l'absence de conventions spécifiques pour noter l'intonation), ce qui entraîne une délimitation de la question, par son bornage à gauche et à droite, différente de l'anglais en espagnol.

Cet exemple pose un problème intéressant concernant la forme "tutone ", traitée par la transcription comme une forme espagnole. On a affaire à une recherche lexicale de $\mathrm{A}$, qui bricole une forme inédite en espagnol (où par ailleurs « tutor » est disponible). On peut se poser la question du statut de cette forme - qui est transcrite ici dans la même police de caractère que ce qui précède et se voit donc traitée comme appartenant au même système linguistique (ce qui pose un problème à la traduction, qui propose une forme anglaise «bricolée »). Cette question n'est pas soulevée dans le texte, qui n'analyse pas cette forme.

41 Le codage typographique des langues oblige donc à trancher nécessairement quant à l'appartenance catégorielle des formes: ceci pose problème, notamment en ce qui concerne des formes potentiellement ambiguës qui peuvent aussi bien appartenir à une langue ou à une autre, ou constituer ce que le linguiste catégorise souvent comme des « interférences» ou bien des «emprunts». Voici comment Poplack traite la plus ou 
moins grande intégration d'une forme dans une autre langue - sur la base de laquelle elle décidera s'il s'agit d'un code-switch ou non :

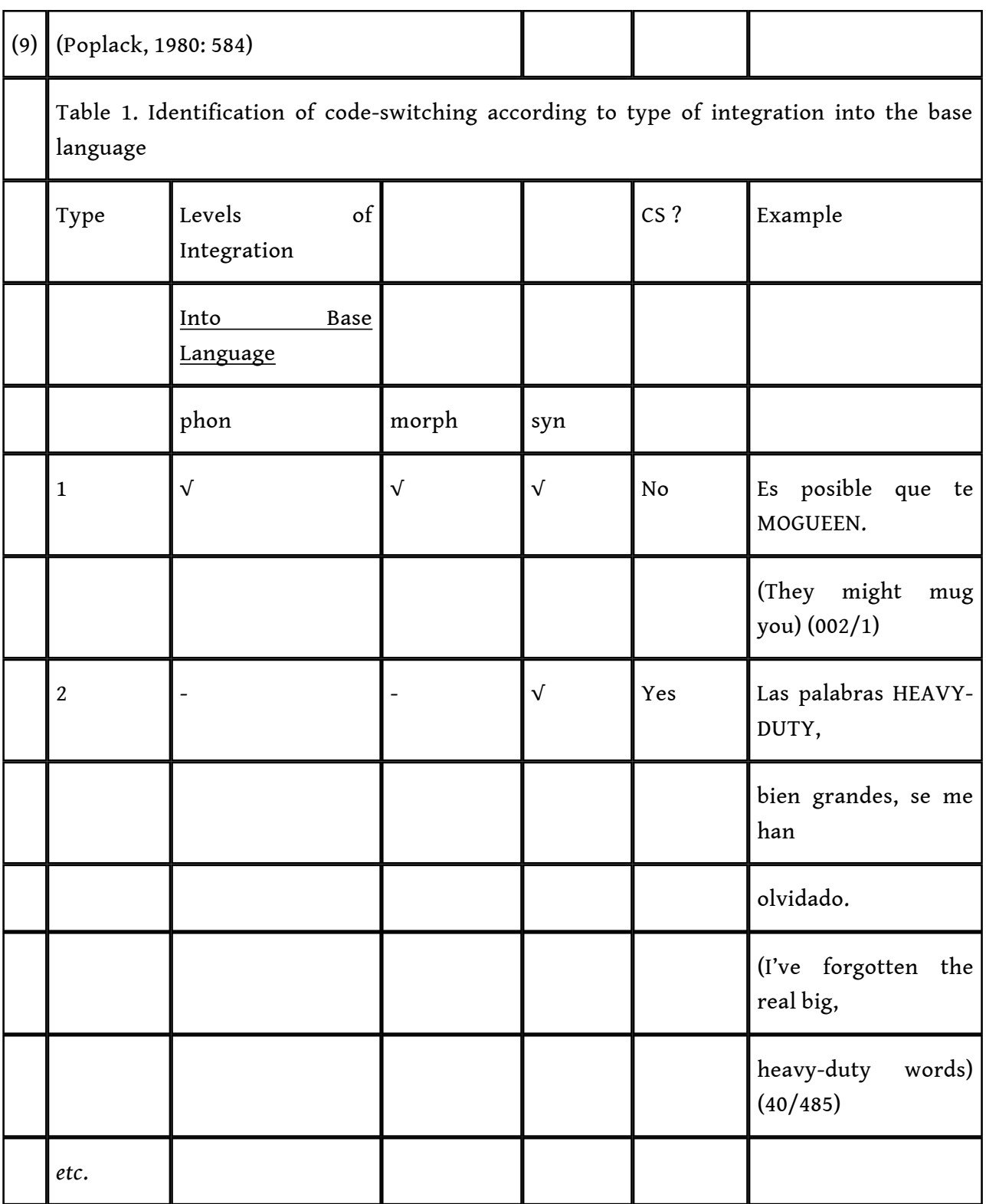

Alors que le tableau vise une catégorisation des formes en termes de code-switch (type 1) ou non (type 2), en reconnaissant leur plus ou moins grande intégration dans la langue de base ; alors que l'analyse note que "the example of type 1, mogueen, is phonologically, morphologically and syntactically integrated into the base language, although etymologically a loan word from English "mug ». It is here considered as instance of monolingual Spanish discourse » (584) - le marquage typographique par contre continue à les traiter comme appartenant à une langue de départ bien déterminée, autre que celle de la langue de base. Le choix des conventions de transcription semble ainsi dicté par la thèse implicite que les langues sont des systèmes autonomes nettement séparables et a comme effet de produire des données conformes à cette thèse. 


\section{Choix de transcription, perspectives des transcripteurs et perspectives des locuteurs}

Cet article a voulu montrer les enjeux de décisions conduisant à incorporer dans la transcription des dimensions théoriquement sensibles qui auraient pu être traitées dans l'analyse. Le transcripteur peut d'une part traiter implicitement certaines dimensions problématiques en résolvant les difficultés qu'elles posent par des choix de transcription qui les intègrent dans la représentation écrite de l'oral, en les naturalisant et les dotant d'un caractère d'évidence objectivée; il peut d'autre part les traiter d'une façon thématisée, qui permet souvent leur discussion dans l'analyse qui suit la transcription.

Cette alternative en introduit une autre : quels sont les observables que la transcription rend disponibles? S'agit-il de dimensions rendues pertinentes par les modèles théoriques et analytiques du chercheur, dont l'importance préexiste aux données particulières transcrites et qui est décidée et validée de façon générale sur la base d'autres corpus empiriques? ou bien s'agit-il de dimensions dont la pertinence est à démontrer sur la base de transcriptions particulières, considérées dans leur spécificité et à travers les procédures de pertinentisation mises en œuvre par les participants eux-mêmes avant que par l'analyste? Alors que la première question renvoie à une approche exogène, étique de la transcription et de son analyse, la seconde renvoie à un traitement endogène, émique, considérant les orientations des participants vers tel ou tel phénomène.

Si la première permet un codage des transcriptions en vue de relevés quantitatifs, ainsi que le traitement des exemples comme confirmation d'hypothèses formulées ailleurs, la seconde est plutôt orientée vers la "découverte» de phénomènes dont la pertinence n'est pas affirmée a priori. Les deux types de transcription n'échappent pas à leur dimension indexicale et interprétative, mais la seconde décide de suspendre certains choix de transcription ou de privilégier une notation relevant de la transcription plutôt que de la description, en considérant qu'il revient à l'analyse de tirer certaines conclusions.

Cette deuxième façon de traiter la transcription va de pair avec une «mentalité analytique " développée en particulier dans le cadre de l'analyse conversationnelle ( $c f$. Psathas \& Anderson, 1990), qui s'intéresse à la façon dont les participants eux-mêmes s'orientent vers un découpage à toutes fins pratiques de la conversation en unités pertinentes par rapport à l'action en cours (Schegloff, 1996; Selting, 1985; Mondada, 2000b) et à la façon dont la valeur d'une ressource linguistique ou sa catégorisation comme appartenant à telle ou telle "grammaire" émerge de son exploitation locale (Auer, 1981, 1995 ; Mondada, 1999b). Tenir compte de l'orientation des membres implique ainsi une redéfinition d'objets classiques en linguistique et stimule un regard particulièrement attentif envers la représentation des données dans les transcriptions : le statut de la transcription est en effet radicalement différent dans ce second cas - la transcription n'étant pas un exemple illustratif parmi d'autres d'un modèle établi par ailleurs, mais un lieu constitutif à partir duquel émergent, dans la pertinence de leurs détails, des phénomènes qui sont traités, problématisés, interprétés par les participants en premier lieu et qui feront l'objet du travail de l'analyste-transcripteur en second lieu ${ }^{19}$. 


\section{BIBLIOGRAPHIE}

ATKINSON, J. M. (1984). Our Masters' Voices : The Language and Body Language of Politics. London : Methuen.

AUER, P. (1981). « Bilingualism as a members' concept : Language choice and language alternation in their relation to lay assessments of competence. » Papiere des Sonderforschungsbereich 99 Linguistik, Universität Konstanz, 54.

AUER, P. (1993). Ueber < =. Zeitschrift für Literaturwissenschaft und Linguistik (Lili), 90/91, 104-138.

AUER, P. (1995). « The pragmatics of code-switching. » In L. Milroy, P. Muysken (Eds.), One Speaker, Two Languages : Cross-disciplinary Perspectives on Codeswitching (115-135). Cambridge : Cambridge University Press.

BERGMANN, J. R. (1985). «Flüchtigkeit und methodische Fixierung sozialer Wirklichkeit : Aufzeichnungen als Daten der interpretativen Soziologie. » In W. Bonss, H. Hartmann (Eds.), Entzauberte Wissenschaft (Sonderband 3 der Sozialen Welt) Göttingen : Otto Schwarz.

BERRENDONNER, A., \& REICHLER-BÉGUELIN, M.-J. (1989). « Décalages : les niveaux de l'analyse linguistique. » Langue Française, 81, 99-125.

BERTHOUD, A.-C., \& MONDADA, L. (Eds.). (2000). Modèles du discours en confrontation. Berne : Lang. BLANCHE-BENVENISTE, C., \& JEANJEAN, C. (1987). Le français parlé. Edition et transcription. Paris : INALF. BRES, J. (1995). « Alors raconte ! », In Véronique, D., Vion, R. (Eds). Des savoir-faire communicationnels, Aix-en-Provence : Presses de Provence.

CHAFE, W. (1993). « Prosodic and functional units of language », In Edwards \& Lampert (Eds.), 33-45.

CHAFE, W. (1994). Discourse, Consciousness and Time : The Flow and Displacement of Consciousness Experience in Speaking and Writing. Chicago : University of Chicago Press.

DAUSENDSCHÖN-GAY, U., GÜLICH, E., \& KRAFFT, U. (1992). « Gemeinsam schreiben. » In H. P. Krings \& G. Antos (Eds.), Textproduktion. Neue Wege der Forschung (219-255). Trier : Wissenschaftlicher Verlag.

DU BoIs, J. W. (1991). « Transcription design principles for spoken discourse research. » Pragmatics, $1,71-106$.

DURANTI, A. (1997). « Transcription : from writing to digitized images. » InLinguistic Anthropology. Cambridge : Cambridge University Press.

EDWARDS, J.A. (1993). « Principles and contrasting systems of discourse transcription. » In Edwards \& Lampert (Eds.), 3-32.

EDWARDS, J. A., \& LAMPERT, M. D. (Eds.). (1993). Talking Data: Transcription and Coding in Discourse Research. Hillsdale : Lawrence Erlbaum.

EHLICH, K., \& REHBEIN, J. (1976). « Halbinterpretative Arbeitstranskriptionen (HIAT). » Linguistische Berichte, 45, 21-41. 
FRANCK, D. (1985). « Das Gespräch in Zeitalter seiner technischen Reproduzierbarkeit. » In E. Gülich, T. Kotschi (Eds.), Grammatik, Konversation, Interaktion. Beiträge zum Romanistentag 1983, Tübingen : Niemeyer.

GOODY, J. (1977). The Domestication of the Savage Mind. Cambridge : CUP.

GUMPERZ, J.J., \& BERENZ, N. (1993). « Transcribing conversational exchanges. » In Edwards \& Lampert (Eds.), 91-122.

HARRIS, R. (1981). The Language Myth. London : Duckworth.

JEFFERSON, G. (1985). « An exercise in the transcription and analysis of laughter. » In T. A. v. Dijk (Ed.), Handbook of Discourse Analysis. Volume 3. New York : Academic Press.

KeLLY, J. \& LOCAL, J.K. (1989). « On the use of general phonetic techniques in handling conversational material. » in P. Bull, D. Roger, (Eds.). Conversation : An Interdisciplinary Perspective. Clevedon : Multilingual Matters.

LATour, B. (1985). « Les “vues” de l'esprit. » Culture Technique, 14, 4-29.

LINELL, P. (1982). The Written Language Bias in Linguistics. Linköping : Dep. of Communication Studies.

LYNCH, M. (1988). "The externalized retina : Selection and mathematization » InThe Visual Documentation of Objects in the Life Sciences. Human Studies, 11, 201-234.

MONDADA, L. (1998). « Technologies et interactions sur le terrain du linguiste. » Le travail du chercheur sur le terrain. Actes du Colloque de Lausanne. Cahiers de l'ILSL, 10, 39-68.

MONDADA, L. (1999a). « L'accomplissement de l'« étrangéité » dans et par l'interaction : procédures de catégorisation des locuteurs. » Langages, 134, 20-34.

MONDADA, L. (1999b). « Alternances de langues et linguistique des pratiques interactionnelles. » Cahiers du Français Contemporain, 5, 83-98.

MONDADA, L. (2000a). « La compétence de catégorisation : procédés situés de catégorisation des ressources linguistiques. » In P. Martinez, S. Pekarek (Eds.), Actes de la Journée d'Etude « Notions en question : les contacts de langues en didactique ». Paris : Didier Erudition.

MONDADA, L. (2000b). « Grammaire-pour-l'interaction et analyse conversationnelle. » In A.-C. Berthoud, L. Mondada (Eds.), Modèles du discours en confrontation Berne : Lang.

MONDADA, L. (2000c). « Pour une approche des activités de catégorisation. » In L. Gajo \& L. Mondada, Interactions et acquisitions en contexte. Fribourg : Editions Universitaires.

MOREL, M.-A., DANON-BOILEAU, L. (1998). Grammaire de l'intonation. L'exemple du français. Paris : Ophrys.

MYERS-SCOTTON, C. (1988). « Codeswitching as indexical of social negotiations. » In M. Heller (Ed.), Code-Switching : Anthropological and Sociolinguistic Perspectives. Berlin : de Gruyter.

O'CONNELL, D.C., KOWAL, S. (1994). « Some current transcription systems for spoken discourse : A critical analysis. » Pragmatics, 4(1), 81-107.

OCHS, E. (1979). « Transcription as theory. » In E. Ochs, B.B. Schieffelin (Eds.), Developmental Pragmatics. New York : Academic Press.

ONG, W. (1982). Orality and Literacy. The Technologizing of the Word. New York : Routledge. 
ORLETTI, F., \& TESTA, R. (1991). « La trascrizione di un corpus di interlingua : Aspetti teorici e metodologici. » Studi Italiani di Linguistica Teorica ed Applicata, 20(2), 243-283.

PERDUE, C. (1982). Second Language Acquisition by Adult Immigrants. A Field Manual. Strasbourg: European Science Foundation.

POPLACK, S. (1980). « Sometimes I'll start a sentence in Spanish y termino en español : Toward a typology of code-switching. » Linguistics, 18, 581-618.

PoyAtos, F. (1993). « The many voices of laughter : A new audible-visual paralinguistic approach. » Semiotica, 93, 62-81.

PSATHAS, G., ANDERSON, T. (1990). « The 'practices' of transcription in Conversation Analysis. » Semiotica, 78(1-2), 75-100.

SACKS, H., SCHEGLOFF, E.A., JEFFERSON, G. (1974). « A simplest systematics for the organization of turn-taking for conversation. » Language, 50, 696-735.

SCHEGLOFF, E.A. (1996). « Turn organization : One intersection of grammar and interaction. » In E. Ochs, E. A. Schegloff, S. A. Thompson (Eds.), Grammar and Interaction. Cambridge : CUP.

SCHMALE, G. (1987). « Reziprozität als Grundlage kooperativen Handelns in Kontaktsituationen zwischen deutschen und französischen Sprechern. » In F. Liedtke, R. Keller (Eds.), Kommunikation und Kooperation. Tübingen: Niemeyer.

ScHWiTALLA, J. (à paraître). « Lächelndes Sprechen und Lachen als Kontextualisierungsverfahren. »In K. Adamzik, H. Christen (Hg.), Festschrift für Gottfried Kolde zum 65. Geburtstag. Tübingen : Niemeyer.

SELTING, M. (1995). « Der “möglische Satz” als interaktiv relevante syntaktische Kategorie. » Linguistische Berichte, 158, 298-325.

SELTING, M., et alii. (1998). « Gesprächsanalytisches Transkriptionssystem (GAT). » Linguistische Berichte, 173, 91-122.

VASSEUR, M.-T. (1993). « Gestion de l'interaction, activités métalangagières et apprentissage en langue étrangère. » AILE, 2, 25-59.

\section{NOTES}

1. De ce point de vue on n'oubliera pas le rôle sélectif exercé d'abord par l'enregistrement - par exemple par le positionnement du micro, par le cadrage de la caméra, par les choix des moments à enregistrer, par la délimitation de leur début et de leur fin, outre les effets provoqués par l'observateur ou ses prothèses techniques. L'enregistrement constitue donc la première opération de sélection, la transcription la seconde. Néanmoins on considère généralement que les «données » sont constituées par l'enregistrement et non par la transcription, qui n'en est qu'une re-présentation heuristique, un support pour l'analyse, laquelle se doit de revenir à la bande originale.

2. Les pratiques effectives de la transcription sont parfois très éloignées des affirmations développées à ce sujet, comme le montrent la division du travail entre producteurs et utilisateurs de transcriptions, attribuant implicitement à la tâche de transcrire un rôle subalterne pouvant être délégué, ainsi que les inconsistances de nombreuses transcriptions publiées, auxquelles contribue souvent le manque de sensibilité des maisons d'édition à ce propos. 
3. De nombreuses discussions présentes dans la littérature insistent sur la dimension « ergonomique » de la transcription, ayant à faire avec sa lisibilité, sa cohérence, sa flexibilité, sa reproductibilité, etc. (voir par exemple Du Bois, 1991; Edwards, 1993; Orletti \& Testa, 1991 : 267sv). Nous n'insisterons pas sur cet aspect bien traité par ailleurs, même si les choix qu'il entraîne peuvent avoir des effets théoriques importants.

4. Le même genre de considérations vaut pour la transcription de la gestualité, que nous ne pourrons toutefois pas prendre en considération ici faute de place.

5. Dans ce sens la réflexion sur la transcription rejoint celle sur la literacy, où des auteurs classiques comme Ong (1982) ou Goody (1977) ont insisté sur les effets du passage à l'écrit. Certains linguistes ont thématisé les conséquences de la literacy sur les modèles linguistiques : $c f$. Linell (1982), Harris (1981).

6. Nous prolongeons ici à propos de la transcription une réflexion en cours sur les activités de catégorisation des linguistes et des locuteurs portant sur les ressources linguistiques qu'ils exploitent à des fins pratiques diverses (Mondada, 1999a, 2000a, 2000c).

7. Voir le volume édité par Berthoud \& Mondada (2000) qui réunit des auteurs explicitant des positions très différentes à ce sujet.

8. Nous avons maintenu dans la mesure du possible la disposition typographique et spatiale des citations que nous donnons en exemple.

9. Bien que parfois on puisse y trouver des commentaires sur les bruits dans la rue, sur la toux d'un interlocuteur ou sur la sonnerie d'un téléphone, qui montrent que d'autres que le locuteur peuvent faire irruption dans cet espace réservé en principe à lui seul.

10. Au contraire, pour une revendication explicite de l'identification du tour et du paragraphe, voir Selting et alii (1998: 97): "Neue Sprecherbeiträge (Turns), die ohne Simultansprechen einsetzen, beginnen mit einer neuen Transkriptzeile. Das Untereinander der Zeilen bildet ikonisch das Nacheinander der Sprecherbeiträge $a b$ ».

11. Sur cette distinction $c f$. Mondada (2000b).

12. La traduction des transcriptions est confrontée à des choix analogues, pouvant faire privilégier le sens global dans une traduction ligne par ligne ou découper plus finement la chaîne parlée dans une traduction morphème à morphème ( $c f$. Duranti, 1997).

13. Les différentes notations possibles et leurs nuances sont rendues visibles dans l'extrait (3) par les deux façons de transcrire «(distinctement) perturbaTION» (116) et «per . tur . ba . TION » (118).

14. Pour d'autres exemples de préférence pour la description sur la transcription, voir le traitement de l'« interruption » - un phénomène dont la définition et les modes d'observabilité sont sujets à controverses en analyse de l'interaction - dans cet extrait de Myers-Scotton (1988 : 177) :

(He est en train de demander à She de danser avec lui)

She (Swahili) : Nimechoka! (I'm tired!)

He (Swahili): Tafadhali (Please)

She (interrupting) (English): Ah, stop bugging me.

15. La notation des « $\mathrm{h}$ » et des « $\mathrm{uh}$ » avec leurs variantes pose de nombreux problèmes, qui sont analysés par O'Connell et Kowal (1994). Poyatos (1993: 64) propose quant à lui jusqu'à 30 variantes phonétiques pour transcrire le rire. $C f$. aussi les transcriptions proposées par Schwitalla (à paraître).

16. Un problème analogue s'est posé à Atkinson (1984) travaillant sur les discours des hommes politiques, pour lesquels il a mis au point un système particulier de notation des applaudissements.

17. Pour une critique et une sophistication de la transcription phonétique, voir Kelly \& Local (1989). 
18. Le terme même de "code-switching " ou de "alternance des langues » renvoie à cette conception.

19. On comprend alors en quoi la transcription devient un moment central de l'analyse ellemême et est donc difficilement délégable à d'autres.

\section{RÉSUMÉS}

Cet article envisage les pratiques de la transcription du point de vue de leurs conséquences théoriques. Il procède pour cela en analysant des extraits de transcription de différents auteurs et de leurs conventions, en se focalisant sur un problème central pour la linguistique de l'oral : le découpage, l'identification, la définition des unités pertinentes de la langue parlée. A travers la façon dont celles-ci sont visualisées, spatialisées, représentées par écrit, l'analyse montre que la transcription matérialise des segmentations très différentes des unités de l'interaction, ainsi que des catégorisations variables des formes linguistiques ou paralinguistique ou des formes pouvant être conçues comme appartenant à une langue ou à une autre. Les enjeux épistémologiques de la transcription reposent précisément sur cette «incorporation » de présupposés théoriques dans les modes de représentation de l'oral.

This article deals with the theoretical consequences of the transcription practices. Detailed analysis of transcript excerpts taken from different authors and of their conventions is provided. It focusses on a central problem within the approaches of spoken language: the segmentation, identification, and definition of relevant units. Describing how they are visualized, spatialized, and represented, the analysis shows that very different models of interactional units and of recognizable linguistic patterns (linguistic forms categorized as belonging to a linguistic system, as being deviant or indetermined; paralinguistic phenomena holistically described or analytically articulated; forms categorized as belonging to a language or another, or categorized as hybrid) are materialized within the transcription choices. The theoretical dimension of the transcription practice is precisely located in this "embodiment" of theoretical presuppositions within the modes of representation of spoken language.

\section{AUTEUR}

\section{LORENZA MONDADA}

Université de Bâle

Romanisches Seminar, Stapfelberg 7/9, CH 4051 Basel

lorenza.mondada@unibas.ch 